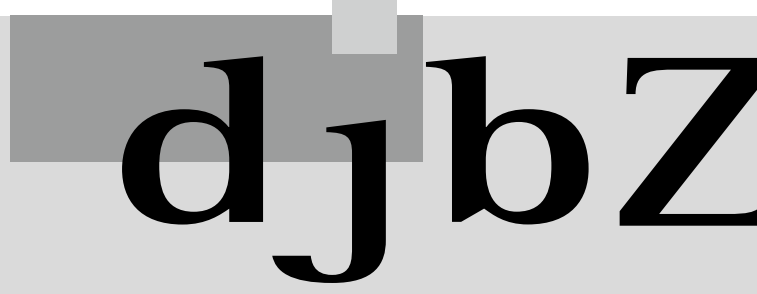

Zeitschrift des

Deutschen

Juristinnenbundes

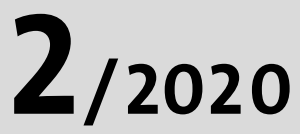

23. Jahrgang, Seite 47-104

Herausgeber: Deutscher Juristinnenbund e. V.

Präsidium: Prof. Dr. Maria Wersig, Hochschullehrerin, Dortmund (Präsidentin); Oriana Corzilius, Rechtsanwältin (Syndikusrechtsanwältin), Frankfurt am Main; Claudia Zimmermann-Schwartz, Ministerialdirigentin a.D., Düsseldorf (Vizepräsidentinnen); Petra Lorenz, Regierungsdirektorin i.R., Sinzheim (Schatzmeisterin); Schriftleitung: Anke Gimbal, Rechtsassessorin, Geschäftsführerin Deutscher Juristinnenbund e.V., Berlin.

\title{
Die Regionalgruppen und der Regionalgruppenbeirat
}

\section{Antje Sedemund-Treiber \\ djb-Ehrenpräsidentin, Bonn}

Ich gratuliere dem Regionalgruppenbeirat und dem djb insgesamt zu dieser Ausgabe der djbZ! Die Regionalgruppen sind, wie es der Vorstand des Regionalgruppenbeirats in seinem Editorial treffend formuliert, die Basis und das Rückgrat des djb. Sich einmal etwas näher mit ihnen und dem Regionalgruppenbeirat zu befassen, ist wichtig, um die innere Struktur unseres Verbandes besser zu verstehen. Ich möchte im Folgenden mit Ihnen ein wenig darüber nachdenken.

\section{Die Vorgeschichte}

Als ich dem djb 1976 beitrat, gab es noch keine Regionalgruppen im heutigen Sinne. In verschiedenen Städten hatten sich interessierte Mitglieder zu örtlichen Gruppierungen zusammengeschlossen. Als Spontangründungen waren diese über die alte Bundesrepublik unregelmäßig verteilt. Eine Struktur auf der Ebene der Bundesländer fehlte ganz. Teilweise überschritt der Einzugsbereich einer örtlichen Gruppe die Grenzen des Bundeslandes, in dem die Gruppe ihren Sitz hatte.

Die Initiative für die Bildung örtlicher Gruppierungen in Düsseldorf, Köln und Bonn geht auf Frau Dr. Peschel-Gutzeit zurück, die in unserem Verband schon damals sehr aktiv war und 1977 auch dessen Leitung übernahm. Im Frühsommer des Jahres 1976 oder 1977 lud Frau Dr. Peschel-Gutzeit die Mitglieder unseres Verbandes aus Nordrhein-Westfalen zu einem Treffen in Düsseldorf ein und gründete eine Untergruppe NordrheinWestfalen des djb. Gleichzeitig regte sie an, sich auch auf lokaler Basis zu organisieren. Frau Dr. Jank übernahm diese Anregung für Düsseldorf, Frau Dr. Hinzen für Köln und ich für Bonn. Diese drei örtlichen Gruppierungen, die sich nach meiner Erinnerung später Ortsgruppen nannten, fanden sich schnell zusammen. Noch vor dem Ende des Jahres, in dem sie sich gebildet hatten, traten wir mit einer gemeinsamen öffentlichen, überraschend gut besuchten Veranstaltung im Bonner Presseclub in das Licht der Öffentlichkeit. Die Gruppe Bonn des djb habe ich nach ihrem Entstehen geleitet, bis ich mich gegen Ende der 1980er Jahre stärker im Bundesvorstand engagierte.

Während meiner Zeit als 1. Vorsitzende des djb (1989 bis 1993) wurde deutlich, dass unser Verband dringend einer besseren Struktur bedurfte. Etwa ab Mitte der 1980er Jahre hatte sich die Zahl unserer Mitglieder fast verdoppelt. Anfang der 1990er Jahre umfasste der djb schon rund 2.000 Mitglieder. Der Bundesvorstand setzte deshalb 1991 eine Arbeitsgruppe ein, die Vorschläge zur Neustrukturierung unseres Verbandes erarbeiten sollte. Sie wurde von den beiden damaligen zweiten Vorsitzenden, Gertrud Hofmann und Christiane Schreiber, geleitet. 1993 legte die Arbeitsgruppe ihre Vorschläge vor. Über eine Satzungsänderung wurden sie umgesetzt und bestimmen die Struktur unseres Verbandes bis heute.

\section{Die gegenwärtige Struktur des djb}

Der djb ist jetzt klar gegliedert: Regionalgruppen bilden die regionale Basis auf kommunaler Ebene, Landesverbände dienen der Zuordnung der Regionalgruppen zu einem Bundesland, auf der Bundesebene ist die Leitung des Gesamtverbandes angesiedelt. Diese drei Ebenen verbindet als vertikale Achse von unten nach oben der Regionalgruppenbeirat.

Der djb umfasst gegenwärtig 36 Regionalgruppen. Sie haben die früheren örtlichen Gruppierungen abgelöst und durch unsere Satzung $(\mathbb{S} 3$ Abs.1,2,3,5) eine einheitliche Rahmenregelung für ihre Gründung, interne Organisation und Finanzierung im Rahmen des Gesamtverbandes erhalten. Ob eine Regionalgruppe entsteht, ist der Initiative der Mitglieder vor Ort überlassen. Deshalb ist ihre Dichte in den einzelnen Bundesländern unterschiedlich.

Die Regionalgruppen haben das Recht, in eigener Zuständigkeit Untergruppen nach räumlichen und fachlichen Ge- 
sichtspunkten zu bilden, die der Gesamtverband mitfinanziert ( $\$ 3$ Abs.3, 5 der Satzung). Fachliche Untergruppen können die Gebiete mehrerer Regionalgruppen umfassen.

Zu einem Landesverband ( $\$ 3$ Abs.1, 4, 5 der Satzung) werden mehrere Regionalgruppen eines Bundeslandes zusammengefasst. Besteht in einem Bundesland nur eine Regionalgruppe, bildet diese zugleich den Landesverband. Das trifft zum Beispiel für die Stadtstaaten Berlin und Hamburg zu. Der Landesverband ist für den djb Ansprechpartner auf der Ebene der Kommunen und einzelnen Bundesländer.

Eine für unseren Verband wesentliche Neuerung war die Einführung der Institution des Regionalgruppenbeirats ( $\$ 12$ der Satzung). Er ist ein eigenständiges Organ unseres Verbandes ( $\mathbb{S} 5$ der Satzung) und vertritt die Regionalgruppen auf der Bundesebene gegenüber dem Bundesvorstand. Sein Vorstand ist Mitglied des Bundesvorstandes und nimmt an dessen Sitzungen teil $(\$ 10$ Abs. 1 der Satzung). Die Aufgaben, die der Regionalgruppenbeirat wahrnimmt ( $\mathbb{1 2}$ Abs. 2 der Satzung), sind wichtig:

- Der Regionalgruppenbeirat gewährleistet den Informationsaustausch

- Er gibt Anregungen und Empfehlungen für die Arbeit des Gesamtverbandes

- Er gibt Empfehlungen für die Höhe der finanziellen Zuweisungen an die Landesverbände, Regionalgruppen und deren Untergliederungen

- Last but not least hat er das Recht, von dem Präsidium und den Vorsitzenden der Kommissionen Bericht zu verlangen

Durch den Regionalgruppenbeirat begegnen sich die Basis unseres Verbandes und dessen Leitung unmittelbar. Die Basis kann Einblick in die Arbeit des Bundesvorstandes und auf diesen Einfluss nehmen. Umgekehrt kann sich die Leitung unseres Verbandes direkt über die Lage an der Basis und deren Arbeit informieren.

\section{Hat sich die gegenwärtige Struktur des djb bewährt?}

Sehen wir uns die verschiedenen Bereiche an:

\section{Die Regionalgruppen}

Sie sind Stätten der Begegnung, des Gesprächs und Erfahrungsaustausches und nicht zuletzt der Geburt von Ideen.

Der juristische Beruf hat zahlreiche und vielschichtige Gesichter. Die Beiträge der Regionalgruppen in diesem Heft der djbZ vermitteln einen Eindruck davon, wie viele und unterschiedliche Fachkompetenzen sich in unserem Verband zusammenfinden. Dieser vielseitigen Expertise und darüber hinaus fundierter Berufserfahrung zu begegnen ist für jedes Mitglied unseres Verbandes schon als solches ein Gewinn und im Übrigen eine wesentliche Grundlage dafür, gemeinsam angemessene Lösungen für anstehende gesellschaftspolitische Probleme zu entwickeln. Hier entstehen Ideen, die über die Arbeit des Gesamtverbandes weitergetragen und umgesetzt werden können.

Leider wird von der Möglichkeit, anstehende Probleme auch einmal in regional übergreifenden Untergruppen zu behandeln, bisher kein Gebrauch gemacht. Eine solche Arbeit würde die Basis bereichern und beleben. Der Umfang der Fachkompetenz und Erfahrung, die eingebracht werden, würde erweitert.
Der fachliche Kontakt in den Regionalgruppen hat aber auch eine wichtige persönliche Komponente. Gleichgesinnte treffen sich. Darüber hinaus sprechen sie - wie es Frau Seeliger in ihrem Beitrag in diesem Heft für die Bonner Regionalgruppe ausführt die gleiche Sprache. In einem solchen Umfeld ergeben sich unkompliziert und schnell auch persönliche Kontakte. Muss ich das näher ausführen? Wir alle haben dies erfahren. Die Treffen gerade der Regionalgruppen führen nicht nur zu interessanten Begegnungen. Aus ihnen haben sich auch wirkliche Freundschaften entwickelt, die ein Leben lang halten und sehr bereichern.

\section{Die Landesverbände}

Während die neuen Regelungen unserer Satzung über die Regionalgruppen an Bestehendes anknüpfen konnten, wurde mit der Einführung der Landesverbände Neuland betreten. Anlass hierfür waren Erfahrungen aus der Praxis, die die Arbeit der früheren örtlichen Gruppen wiederholt erschwert hatten.

Verschiedene der früheren regionalen Gruppen hatten sich an rechtspolitischen Vorhaben auf kommunaler Ebene oder der Ebene eines Bundeslandes beteiligt oder wollten dies tun, stießen aber teilweise auf Schwierigkeiten. Ein anschauliches Beispiel hat mir Christiane Schreiber, eine der Mütter unserer modernisierten Satzung, geschildert. Als Leiterin der damaligen örtlichen Gruppe für Saarbrücken und Trier wollte sie einmal gegenüber dem Landesfrauenrat des Saarlandes eine Stellungnahme abgeben. Dies war ihr als Leiterin nur einer örtlichen Gruppe unseres Verbandes nicht möglich. Die von dem Frauenrat auf Bundesebene unabhängigen Landesfrauenräte sind selbständig organisiert und haben eigene Satzungen. Der Landesfrauenrat des Saarlandes verlangte von einem mitwirkenden Verband eine Organisationseinheit auf der Ebene eines Bundeslandes. Sie ist mit den Landesverbänden unserer Satzung geschaffen worden. Auf dieser Basis hat sich Christiane Schreiber dann nicht nur Gehör in dem Landesfrauenrat des Saarlandes verschaffen können. Sie hat ihn darüber hinaus später Jahre lang geleitet.

Die Mitarbeit auf kommunaler Ebene oder der Ebene eines Bundeslandes ist im Übrigen ein wichtiger Weg für unseren Verband, sich über die Bundesebene hinaus in das rechtspolitische Geschehen einzubringen. Sie eröffnet zudem solchen Mitgliedern die Möglichkeit einer aktiven gesellschaftspolitischen Mitwirkung, die sich aus persönlichen Gründen nicht in der Lage sehen, an der zeitaufwendigeren Arbeit unseres Verbandes auf Bundesebene, etwa in einer Kommission, mitzuwirken.

Die Aktivitäten des djb auf kommunaler Ebene und auf der Ebene einzelner Bundesländer waren in unserem Gesamtverband wenig bekannt. Das vorliegende Heft zeigt, dass in einigen Regionen bereits lebhafte Kontakte bestehen. In einem späteren Heft der djbZ könnten wir hierüber vielleicht einmal mehr erfahren.

\section{Der Regionalgruppenbeirat}

Auch mit dem Institut des Regionalgruppenbeirats hat unsere Satzung etwas grundlegend Neues für unseren Verband geschaffen. Seine Einführung ist die Antwort unserer Satzung auf die wichtige Frage, wie in dem damals stark gewachsenen und hoffentlich weiterwachsenden djb eine gut funktionierende und angemessene 
Verbindung zwischen der Basis und ihrer Leitung, bei uns dem Bundesvorstand, hergestellt und erhalten werden kann. Einfach formuliert geht es um die Verbindung von „unten“ nach „oben“ und umgekehrt. Um als starke vertikale Achse fungieren zu könne, ist der Regionalgruppenbeirat hierfür mit beachtlichen Rechten, aber auch Pflichten ausgestattet worden. Schauen wir uns die wichtigsten an:

Der Regionalgruppenbeirat gewährleistet den Informationsaustausch ( $\mathbb{S} 12$ Abs. 2 der Satzung). Der Bundesvorstand einerseits und anderseits die Basis müssen wissen, was in unserem Verband abläuft, um effektiv arbeiten und zusammenwirken zu können. Von grundlegender Bedeutung hierfür ist eine gute gegenseitige Information.

Die Information von oben nach unten ist in erster Linie Aufgabe des Bundesvorstandes. Er leistet diese Informationen vor allem durch die djbZ und regelmäßige Newsletter der Präsidentin. Darüber hinaus kann sich der Bundesvorstand natürlich auch in anderer Form mit der Basis in Verbindung setzen. Bewährte Möglichkeiten sind die Teilnahme der Präsidentin oder auch der Vorsitzenden einer Kommission an Treffen der Regionalgruppen.

In die Informationskette von oben nach unten ist aber auch der Regionalgruppenbeirat eingebunden. Auf Grund seiner Verpflichtung zur Gewährleistung des Informationsaustausches ist er angehalten, die Basis über die Arbeit und Pläne des Bundesvorstandes zu unterrichten. Das Wort „Austausch“ macht deutlich, dass es hier um eine Information in beide Richtungen geht, also nicht nur von unten nach oben, sondern auch umgekehrt.

Eine Informationskette von unten nach oben zu schaffen, ist in erster Linie Aufgabe des Regionalgruppenbeirates. Der Regionalgruppenbeirat hat deshalb über seinen Vorstand den Bundesvorstand über die Befindlichkeiten, Vorstellungen und Ideen der Basis zu unterrichten, damit der Bundesvorstand diese bei seiner Arbeit berücksichtigen kann.
Der Regionalgruppenbeirat hat weiter Anregungen und Empfehlungen für die Verbandsarbeit zu geben ( $\mathbb{1} 12$ Abs. 2 der Satzung). Hier geht es um den Kontakt von unten nach oben. Die Basis hat die Möglichkeit, sich auf einem organisiertem Weg Gehör auf Bundesebene zu verschaffen. Erforderliche Abstimmungen unterschiedlicher Auffassungen der Regionalgruppen kann der Vorstand des Regionalgruppenbeirats organisieren.

Schließlich kann der Regionalgruppenbeirat über die ihm obliegenden Empfehlungen für die finanziellen Zuwendungen an die Regionalgruppen, deren Untergruppen sowie an die Landesverbände versuchen, auf die finanzielle Ausstattung der Untergliederungen unseres Verbandes Einfluss zu nehmen. Diese ist für Art und Umfang der Arbeit vor Ort sehr wichtig.

Zusammenfassend bewertet ist der über seinen Vorstand im Bundesvorstand vertretene Regionalgruppenbeirat ein außerordentlich wichtiges Organ unseres Verbandes: Er ist das Bindeglied und Scharnier zwischen der Basis und deren Leitung auf der Bundesebene.

\section{Fazit}

Die organisatorischen Voraussetzungen für ein gutes Miteinander und eine gelingende Zusammenarbeit wurden in der Vergangenheit mit Blick auf die gewachsenen Anforderungen geschaffen, die sich einem größer werdenden Verband stellen. Die immer neue Aufgabe ist es, diesen Rahmen zu nutzen und zu füllen. Da die Facharbeit in unserem Verband ehrenamtlich geleistet wird, ist dies eine spürbare Belastung. Sicherlich wäre es gut, wenn alle Tage doppelt so lang wären, wie sie es tatsächlich sind. Leider bietet unsere Satzung dafür keine Lösung an. Auch ich selbst kann Ihnen mit einem Rat nicht helfen. Aber ich kann Ihnen aus eigener Erfahrung sagen: Es lohnt sich mitzumachen, auf welcher Ebene auch immer. Sie werden dafür reich beschenkt.

\title{
Landesverband Baden-Württemberg / Regionalgruppe Freiburg
}

\author{
Interview mit Dr. Herta Mattes, ehemaliges Vorstandsmitglied und langjährigstes Mitglied der \\ Regionalgruppe Freiburg
}

\section{Petra Lorenz}

Mitglied des Bundesvorstands, Schatzmeisterin des djb und Schriftführerin der Regionalgruppe Freiburg

\section{Anneliese Schmid-Kaufhold}

Vorsitzende der Regionalgruppe Freiburg und Vorstandsmitglied des Landesverbands Baden-Württemberg

Die Regionalgruppe Freiburg hat 60 Mitglieder und trifft sich regelmäßig zum Erfahrungsaustausch oder auch zu besonderen
Themen (Beispiel: djbZ 1-2019, S.33ff). Ein in jeder Hinsicht besonderes Mitglied in unserer Gruppe ist Frau Dr. Herta Mattes: Wir erinnern uns gerne an eine Reihe von Vorträgen, mit denen sie unsere Arbeit inspiriert hat. So zum Beispiel ihren Vortrag im April 2008 über die langjährigen Erfahrungen, Veränderungen und Entwicklungen innerhalb der Justiz seit Ende der 1950er Jahre, welcher uns noch sehr lebhaft und eindrücklich in Erinnerung geblieben ist.

Ebenso hat Frau Dr. Mattes brillante Vorträge gehalten zu den Themen „60 Jahre Grundgesetz“ (2009), „60 Jahre Bundes- 